(C) by Oldenbourg Wissenschaftsverlag, München

\title{
Crystal structure of (1,8-diethyl-1,3,4,9-tetrahydro-pyrano[3,4-b]indol- 1-yl)acetic acid (2-hydroxy-benzylidene)hydrazide, $\mathrm{C}_{24} \mathrm{H}_{27} \mathrm{~N}_{3} \mathrm{O}_{3}$
}

\section{Q.-B. Song* and J. Zhang}

Zhejiang University of Technology, College of Chemical Engineering and Materials Science, State Key Laboratory Breeding Base of Green ChemistrySynthesis Technology, Hangzhou 310014, P. R. China

Received May 22, 2006, accepted and available on-line July 27, 2006; CCDC no. 1267/1791

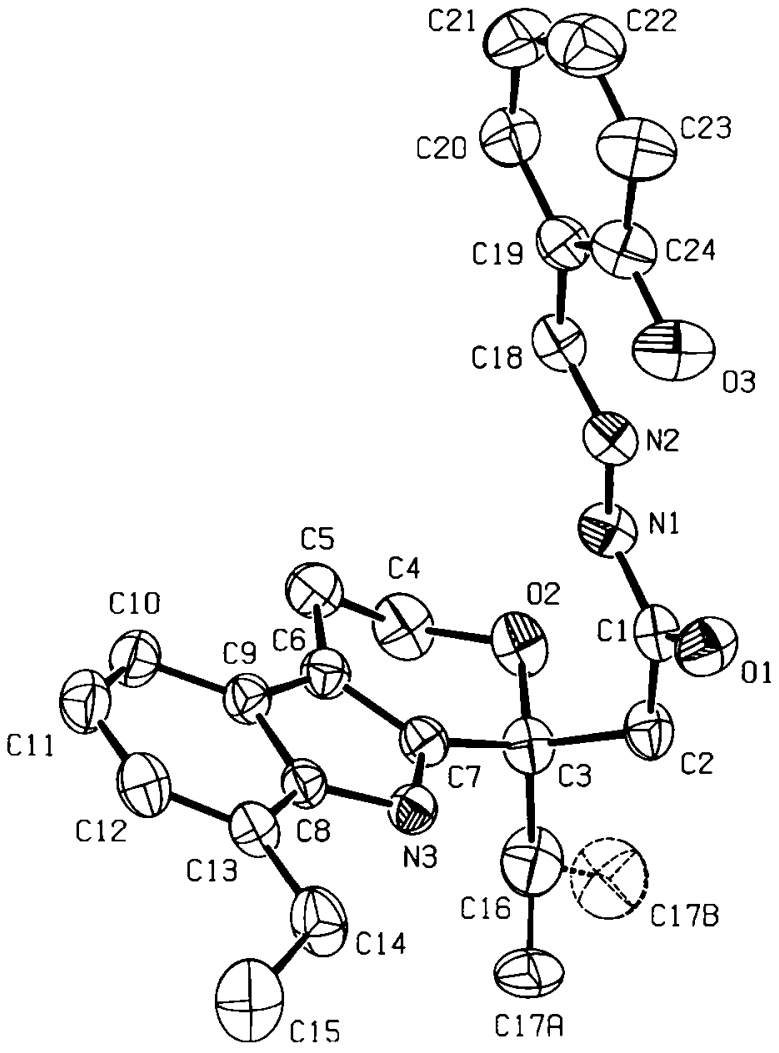

Abstract

$\mathrm{C}_{24} \mathrm{H}_{27} \mathrm{~N}_{3} \mathrm{O}_{3}$, monoclinic, $P 121 / c 1$ (no. 14), $a=11.376(1) \AA$, $b=15.955(2) \AA, c=12.254(1) \AA, \beta=97.352(2)^{\circ}$,

$V=2205.8 \AA^{3}, Z=4, R_{\mathrm{gt}}(F)=0.051, w R_{\mathrm{ref}}\left(F^{2}\right)=0.152$,

$T=293 \mathrm{~K}$

\section{Source of material}

The compound, (1,8-diethyl-1,3,4,9-tetrahydro-pyrano[3,4-b]indol-1-yl)acetic acid (2-hydroxy-benzylidene)hydrazide, is obtained from the condensation reaction of $(1,8$-diethyl-1,3,4,9tetrahydro-pyrano[3,4-b]indol-1-yl)-acetic acid hydrazide with salicylaldehyde [1,2]. A mixture of $1 \mathrm{~g}(3.3 \mathrm{mmol})$ of (1,8-diethyl-1,3,4,9-tetrahydro-pyrano[3,4-b]indol-1-yl)-acetic acid hydrazide, $0.41 \mathrm{~g}(3.32 \mathrm{mmol})$ of salicylaldehyde, $50 \mathrm{~mL}$ of anhydrous ethanol was vigorously stirred at reflux for 1.5 hours. Then the reaction mixture was filtrated and washed with $30 \mathrm{ml}$ ethanol to give $1.21 \mathrm{~g}$ of the title compound (yield $89.9 \%$ ). The crystals were obtained from a solution of the compound in anhydrous ethanol after two days at room temperature (m.p. $462-463 \mathrm{~K}$ ).

\section{Experimental details}

The positions of $\mathrm{H} 1$ (at N1), $\mathrm{H} 2$ (at N3) and $\mathrm{H} 3$ (at $\mathrm{O3}$ ) were found from Fourier difference maps and refined freely. The other $\mathrm{H}$ atoms were placed in calculated positions and allowed to ride on their parent atoms at distances of $0.93 \AA-0.97 \AA$ for $\mathbf{C}-\mathrm{H}$. Isotropic displacement parameters were set at 1.2 to 1.5 times $U_{\text {eq }}$ of the parent atom.

One ethyl group was found to be disordered over two positions caused by rotation around the $\mathrm{C} 3-\mathrm{C} 16$ single bond with approximately equal occupancies. The $\mathrm{C} 17$ atom was split into two positions and refined anisotropically. The corresponding $\mathrm{H}$ atoms at $\mathrm{C} 16$ and $\mathrm{C17}$ were added geometrically in accord with the C16 $-\mathrm{C} 17 \mathrm{~A}$ and $\mathrm{C16}-\mathrm{C} 17 \mathrm{~B}$ bonds.

\section{Discussion}

The title compound is a derivative of the non-steroidal antiinflammatory drugs (NSAIDs) etodolac [3]. Etodolac is an indoleacetic acid derivative with analgesic and limited antiinflammatory properties. Etodolac is rapidly absorbed from the gastrointestinal tract, with a bioavailability of approximately $80 \%$. Like other NSAIDs, etodolac is highly (more than $99 \%$ ) bound to plasma proteins and extensively metabolized in the ljer, with a half-life of approximately six to seven bours [4]. Also, gastrointestinal effect is the most common adverse reactions associated with etodolac [5].

In the title crystal structure, the molecules are interlinked by intermolecular hydrogen bonds N3-HA $\cdots 01$ and $\mathrm{O} 1-\mathrm{HA} \cdots \mathrm{N} 3$ bonds into centrosymmetric dimers. There are two intramolecular hydrogen bonds $\mathrm{O} 3-\mathrm{HA} \cdots \mathrm{N} 2, \mathrm{O} 2-\mathrm{HA} \cdots \mathrm{N} 1$ forming two closed sixmembered loops in each molecule. The phenyl ring and the pyrrole ring are nearly coplanar. In phenyl ring part, the maximum $\mathrm{C}-\mathrm{C}$ bond length is $1.406(3) \AA$, the minimum is $1.363(3) \AA$, and the average $\mathrm{C}-\mathrm{C}$ bond length for the phenyl ring is $1.389(3) \AA$. In the pyrrole ring part, the longer $C-C$ bond is $1.406(3) \AA$ and the other is $1.356(3) \AA$. The pyran ring has a chair form configuration. Other bond lengths and angles are in normal range.

Table 1. Data collection and handling.

\section{Crystal:}

Wavelength:

$\mu$ :

Diffractometer, scan mode:

$2 \theta_{\max }:$

$N(h k l)_{\text {measured, }} N(h k l)_{\text {unique: }}$

Criterion for $I_{\mathrm{obs}}, N(h k l)_{\mathrm{gt}}$ :

$N(\text { param })_{\text {refined: }}$

Programs: colorless, prismatic, size $0.234 \times 0.417 \times 0.498 \mathrm{~mm}$ Mo $K_{\alpha}$ radiation $(0.71073 \AA)$ $0.81 \mathrm{~cm}^{-1}$

Bruker SMART CCD, $\varphi / \omega$ $54^{\circ}$ 12839,4815

$I_{\mathrm{obs}}>2 \sigma\left(I_{\mathrm{obs}}\right), 2251$

294

SHELXS-97 [6], SHELXL-97 [7]

\footnotetext{
* Correspondence author (e-mail: qbsong6@163.com)
} 
Table 2. Atomic coordinates and displacement parameters (in $\AA^{2}$ ).

\begin{tabular}{lllllll}
\hline Atom & Site & Occ. & \multicolumn{1}{l}{$\boldsymbol{x}$} & $y$ & $z$ & $U_{\text {iso }}$ \\
\hline H(1) & $4 e$ & & $0.327(2)$ & $0.187(1)$ & $0.145(2)$ & $0.083(8)$ \\
H(2) & $4 e$ & $0.664(2)$ & $0.072(1)$ & $0.080(2)$ & $0.065(7)$ \\
H(3) & $4 e$ & $0.206(3)$ & $0.184(2)$ & $-0.154(3)$ & $0.12(1)$ \\
H(2A) & $4 e$ & 0.4760 & -0.0043 & 0.1386 & 0.076 \\
H(2B) & $4 e$ & 0.3725 & 0.0174 & 0.2060 & 0.076 \\
H(4A) & $4 e$ & 0.5636 & 0.1991 & 0.4032 & 0.095 \\
H(4B) & $4 e$ & 0.4429 & 0.2477 & 0.3961 & 0.095 \\
H(5A) & $4 e$ & 0.4753 & 0.3124 & 0.2358 & 0.084 \\
H(5B) & $4 e$ & 0.5940 & 0.3223 & 0.3161 & 0.084 \\
H(10) & $4 e$ & 0.7198 & 0.3871 & 0.1402 & 0.081 \\
H(11) & $4 e$ & 0.8481 & 0.3938 & 0.0108 & 0.093 \\
H(12) & $4 e$ & 0.8966 & 0.2765 & -0.0817 & 0.090 \\
H(14A) & $4 e$ & 0.8286 & 0.0686 & -0.0164 & 0.105 \\
H(14B) & $4 e$ & 0.7704 & 0.1045 & -0.1290 & 0.105 \\
H(15A) & $4 e$ & 0.9457 & 0.1531 & -0.1651 & 0.133 \\
H(15B) & $4 e$ & 0.9552 & 0.0570 & -0.1382 & 0.133 \\
& & & & & \\
\hline
\end{tabular}

Table 2. Continued.

\begin{tabular}{lllllll}
\hline Atom & Site & Occ. & \multicolumn{1}{l}{$x$} & \multicolumn{1}{l}{$y$} & \multicolumn{1}{l}{$z$} & $U_{\text {iso }}$ \\
\hline H(15C) & $4 e$ & & 1.0058 & 0.1229 & -0.0495 & 0.133 \\
H(16A) & $4 e$ & 0.5 & 0.6241 & 0.0762 & 0.3850 & 0.110 \\
H(16B) & $4 e$ & 0.5 & 0.5326 & 0.0043 & 0.3585 & 0.110 \\
H(16C) & $4 e$ & 0.5 & 0.6320 & 0.0011 & 0.2854 & 0.110 \\
H(16D) & $4 e$ & 0.5 & 0.6471 & 0.0754 & 0.3682 & 0.110 \\
H(17A) & $4 e$ & 0.5 & 0.6991 & -0.0521 & 0.3587 & 0.125 \\
H(17B) & $4 e$ & 0.5 & 0.7468 & 0.0201 & 0.2895 & 0.125 \\
H(17C) & $4 e$ & 0.5 & 0.6516 & -0.0427 & 0.2335 & 0.125 \\
H(17D) & $4 e$ & 0.5 & 0.4934 & -0.0517 & 0.3655 & 0.130 \\
H(17E) & $4 e$ & 0.5 & 0.4879 & 0.0281 & 0.4393 & 0.130 \\
H(17F) & $4 e$ & 0.5 & 0.6002 & -0.0297 & 0.4549 & 0.130 \\
H(18) & $4 e$ & & 0.2336 & 0.2987 & 0.0764 & 0.074 \\
H(20) & $4 e$ & & 0.1257 & 0.4226 & 0.0017 & 0.098 \\
H(21) & $4 e$ & & 0.0116 & 0.4934 & -0.1365 & 0.124 \\
H(22) & $4 e$ & & -0.0394 & 0.4270 & -0.3052 & 0.133 \\
H(23) & $4 e$ & & 0.0385 & 0.2995 & -0.3386 & 0.116 \\
& & & & & & \\
\hline
\end{tabular}

Table 3. Atomic coordinates and displacement parameters (in $\AA^{2}$ ).

\begin{tabular}{|c|c|c|c|c|c|c|c|c|c|c|c|}
\hline Atom & Site & Occ. & $x$ & $y$ & $z$ & $U_{11}$ & $U_{22}$ & $U_{33}$ & $U_{12}$ & $U_{13}$ & $U_{23}$ \\
\hline$O(1)$ & $4 e$ & & $0.3528(1)$ & $0.05026(9)$ & $-0.0267(1)$ & $0.095(1)$ & $0.070(1)$ & $0.061(1)$ & $0.0046(8)$ & $-0.0036(8)$ & $-0.0128(8)$ \\
\hline$O(2)$ & $4 e$ & & $0.4382(1)$ & $0.1511(1)$ & $0.2945(1)$ & $0.065(1)$ & $0.095(1)$ & $0.0571(9)$ & $-0.0124(8)$ & $0.0161(7)$ & $-0.0125(8)$ \\
\hline$O(3)$ & $4 e$ & & $0.1751(2)$ & $0.2085(1)$ & $-0.2147(2)$ & $0.112(2)$ & $0.117(2)$ & $0.055(1)$ & $0.036(1)$ & $0.005(1)$ & $-0.014(1)$ \\
\hline $\mathbf{N}(1)$ & $4 e$ & & $0.3179(2)$ & $0.1590(1)$ & $0.0807(2)$ & $0.063(1)$ & $0.077(1)$ & $0.045(1)$ & $0.005(1)$ & $0.0020(9)$ & $-0.009(1)$ \\
\hline $\mathbf{N}(2)$ & $4 e$ & & $0.2583(2)$ & $0.2007(1)$ & $-0.0074(1)$ & $0.054(1)$ & $0.073(1)$ & $0.052(1)$ & $0.0008(9)$ & $0.0076(9)$ & $-0.0049(9)$ \\
\hline$N(3)$ & $4 e$ & & $0.6602(1)$ & $0.1232(1)$ & $0.1040(1)$ & $0.053(1)$ & $0.052(1)$ & $0.057(1)$ & $-0.0013(9)$ & $0.0059(8)$ & $-0.0065(9)$ \\
\hline$C(1)$ & $4 e$ & & $0.3647(2)$ & $0.0833(1)$ & $0.0641(2)$ & $0.054(1)$ & $0.062(2)$ & $0.056(1)$ & $-0.014(1)$ & $0.006(1)$ & $-0.004(1)$ \\
\hline$C(2)$ & $4 e$ & & $0.4305(2)$ & $0.0416(1)$ & $0.1636(2)$ & $0.063(1)$ & $0.067(1)$ & $0.059(1)$ & $-0.014(1)$ & $0.007(1)$ & $0.005(1)$ \\
\hline$C(3)$ & $4 e$ & & $0.5150(2)$ & $0.0964(1)$ & $0.2414(2)$ & $0.063(1)$ & $0.068(1)$ & $0.049(1)$ & $-0.008(1)$ & $0.005(1)$ & $0.002(1)$ \\
\hline$C(4)$ & $4 e$ & & $0.4973(2)$ & $0.2200(2)$ & $0.3530(2)$ & $0.079(2)$ & $0.101(2)$ & $0.060(1)$ & $-0.007(1)$ & $0.015(1)$ & $-0.023(1)$ \\
\hline$C(5)$ & $4 e$ & & $0.5415(2)$ & $0.2821(1)$ & $0.2753(2)$ & $0.066(1)$ & $0.074(2)$ & $0.070(2)$ & $0.003(1)$ & $0.007(1)$ & $-0.017(1)$ \\
\hline$C(6)$ & $4 e$ & & $0.6063(2)$ & $0.2351(1)$ & $0.1963(2)$ & $0.054(1)$ & $0.058(1)$ & $0.054(1)$ & $-0.002(1)$ & $0.002(1)$ & $-0.008(1)$ \\
\hline$C(7)$ & $4 e$ & & $0.5916(2)$ & $0.1514(1)$ & $0.1816(2)$ & $0.050(1)$ & $0.059(1)$ & $0.047(1)$ & $-0.003(1)$ & $0.0012(9)$ & $-0.003(1)$ \\
\hline$C(8)$ & $4 e$ & & $0.7203(2)$ & $0.1909(1)$ & $0.0680(2)$ & $0.045(1)$ & $0.053(1)$ & $0.053(1)$ & $-0.0056(9)$ & $0.000(1)$ & $0.001(1)$ \\
\hline$C(9)$ & $4 e$ & & $0.6882(2)$ & $0.2620(1)$ & $0.1251(2)$ & $0.053(1)$ & $0.054(1)$ & $0.054(1)$ & $-0.004(1)$ & $-0.001(1)$ & $-0.002(1)$ \\
\hline$C(10)$ & $4 e$ & & $0.7383(2)$ & $0.3390(1)$ & $0.1031(2)$ & $0.072(2)$ & $0.056(1)$ & $0.072(2)$ & $-0.008(1)$ & $0.003(1)$ & $-0.003(1)$ \\
\hline $\mathrm{C}(11)$ & $4 e$ & & $0.8149(2)$ & $0.3426(2)$ & $0.0262(2)$ & $0.077(2)$ & $0.069(2)$ & $0.086(2)$ & $-0.019(1)$ & $0.011(1)$ & $0.005(1)$ \\
\hline$C(12)$ & $4 e$ & & $0.8442(2)$ & $0.2713(2)$ & $-0.0297(2)$ & $0.066(2)$ & $0.088(2)$ & $0.074(2)$ & $-0.017(1)$ & $0.017(1)$ & $0.002(1)$ \\
\hline$C(13)$ & $4 e$ & & $0.7990(2)$ & $0.1932(1)$ & $-0.0112(2)$ & $0.051(1)$ & $0.071(2)$ & $0.062(1)$ & $-0.008(1)$ & $0.008(1)$ & $-0.008(1)$ \\
\hline C(14) & $4 e$ & & $0.8314(2)$ & $0.1147(2)$ & $-0.0676(2)$ & $0.070(2)$ & $0.101(2)$ & $0.096(2)$ & $-0.017(1)$ & $0.033(1)$ & $-0.029(2)$ \\
\hline$C(15)$ & $4 e$ & & $0.9438(4)$ & $0.1117(3)$ & $-0.1085(4)$ & $0.114(3)$ & $0.120(4)$ & $0.148(6)$ & $-0.017(3)$ & $0.088(4)$ & $-0.081(4)$ \\
\hline$C(16)$ & $4 e$ & & $0.5886(3)$ & $0.0397(2)$ & $0.3266(2)$ & $0.109(2)$ & $0.092(2)$ & $0.065(2)$ & $-0.017(2)$ & $-0.020(2)$ & $0.015(2)$ \\
\hline$C(17 A)$ & $4 e$ & 0.5 & $0.6785(5)$ & $-0.0129(3)$ & $0.3000(4)$ & $0.080(3)$ & $0.081(3)$ & $0.085(3)$ & $0.023(3)$ & $-0.010(3)$ & $0.025(3)$ \\
\hline C(17B) & $4 e$ & 0.5 & $0.5388(6)$ & $-0.0069(4)$ & $0.4022(5)$ & $0.135(5)$ & $0.137(5)$ & $0.094(4)$ & $-0.013(4)$ & $0.032(4)$ & $0.039(4)$ \\
\hline$C(18)$ & $4 e$ & & $0.2198(2)$ & $0.2738(2)$ & $0.0073(2)$ & $0.056(1)$ & $0.077(2)$ & $0.054(1)$ & $-0.005(1)$ & $0.011(1)$ & $-0.010(1)$ \\
\hline$C(19)$ & $4 e$ & & $0.1539(2)$ & $0.3188(1)$ & $-0.0843(2)$ & $0.055(1)$ & $0.071(2)$ & $0.065(2)$ & $-0.002(1)$ & $0.014(1)$ & $0.001(1)$ \\
\hline$C(20)$ & $4 e$ & & $0.1085(2)$ & $0.3977(2)$ & $-0.0671(2)$ & $0.078(2)$ & $0.077(2)$ & $0.092(2)$ & $0.001(1)$ & $0.013(2)$ & $-0.003(2)$ \\
\hline$C(21)$ & $4 e$ & & $0.0393(3)$ & $0.4398(2)$ & $-0.1486(3)$ & $0.108(2)$ & $0.077(2)$ & $0.124(3)$ & $0.014(2)$ & $0.012(2)$ & $0.018(2)$ \\
\hline$C(22)$ & $4 e$ & & $0.0113(3)$ & $0.4006(2)$ & $-0.2502(3)$ & $0.104(2)$ & $0.122(3)$ & $0.104(3)$ & $0.018(2)$ & $0.011(2)$ & $0.041(2)$ \\
\hline$C(23)$ & $4 e$ & & $0.0569(3)$ & $0.3243(2)$ & $-0.2698(2)$ & $0.103(2)$ & $0.124(3)$ & $0.063(2)$ & $0.023(2)$ & $0.008(2)$ & $0.013(2)$ \\
\hline$C(24)$ & $4 e$ & & $0.1303(2)$ & $0.2834(2)$ & $-0.1891(2)$ & $0.072(2)$ & $0.093(2)$ & $0.057(1)$ & $0.008(1)$ & $0.017(1)$ & $0.004(1)$ \\
\hline
\end{tabular}

\section{References}

1. Kucukguzel, S. G.; Mazi, A.; Sahin, F.; Ozturk, S.; Stables, J.: Synthesis and biological activities of diflunisal hydrazide-hydrazones. Eur. J. Med. Chem. 38 (2003) 1005-1013.

2. Ainscough, E.W.; Brodie, A. M.; Dobbs, A. J.; Ranford, J. D.; Waters, J. M.: Antitumour copper(II) salicylaldehyde benzoylhydrazone $\left(\mathrm{H}_{2} \mathrm{sb}\right)$ complexes: physicochemical properties and the single-crystal $\mathrm{X}$-ray structures of $\left[\left\{\mathrm{Cu}\left(\mathrm{H}_{2} \mathrm{sb}\right)\left(\mathrm{CCl}_{3} \mathrm{CO}_{2}\right)_{2}\right\}_{2}\right]$ and $\left[\left\{\mathrm{Cu}(\mathrm{Hsb})\left(\mathrm{ClO}_{4}\right)\left(\mathrm{C}_{2} \mathrm{H}_{5} \mathrm{OH}\right)\right\}_{2}\right]$ and the related salicylaldehyde acetylhydrazone $\left(\mathrm{H}_{2} \mathrm{sa}\right)$ complex, $\left[\mathrm{Cu}(\mathrm{Hsa}) \mathrm{Cl}\left(\mathrm{H}_{2} \mathrm{O}\right)\right] \cdot \mathrm{H}_{2} \mathrm{O}$. Inorg. Chim. Acta 267 (1998) 27-38.

3. Humber, L. G.: Etodolac: the chemistry, pharmacology, metabolic disposition, and clinical profile of a novel anti-inflammatory pyranocarboxylic acid. Med. Res. Rev. 7 (1987) 1-28.
4. Lynch, S.; Brogden, R. N.: Etodolac. A preliminary review of its pharmacodynamic activity and therapeutic use. Drugs 31 (1986) 288-300.

5. Schattenkirchner, $M$ : An updated safety profile of etodolac in several thousand patients. Eur. J. Rheumatol. Inflamm. 10 (1990) 56-65.

6. Sheldrick, G. M.: Phase Annealing in SHELX-90: Direct Methods for Larger Structures. Acta Crystallogr. A46 (1990) 467-473.

7. Sheldrick, G. M.: SHELXI-97. Program for the Refinement of Crystal Structures. University of Göttingen, Germany 1997. 